\title{
Simulation of electron postacceleration in a two-stage laser wakefield accelerator
}

\author{
A. J. W. Reitsma, ${ }^{1,2}$ W. P. Leemans, ${ }^{2}$ E. Esarey, ${ }^{2}$ C. B. Schroeder, ${ }^{2}$ L. P. J. Kamp, ${ }^{1}$ and T. J. Schep ${ }^{1}$ \\ ${ }^{1}$ Technische Universiteit Eindhoven, P.O. Box 513, 5600 MB Eindhoven, The Netherlands \\ ${ }^{2}$ Lawrence Berkeley National Laboratory, Berkeley, California 94720
}

(Received 15 February 2002; published 28 May 2002)

\begin{abstract}
Electron bunches produced in self-modulated laser wakefield experiments usually have a broad energy distribution, with most electrons at low energy (1-3 MeV) and only a small fraction at high energy. We propose and investigate further acceleration of such bunches in a channel-guided resonant laser wakefield accelerator. Two-dimensional simulations with and without the effects of self-consistent beam loading are performed and compared. These results indicate that it is possible to trap about $40 \%$ of the injected bunch charge and accelerate this fraction to an average energy of about $50 \mathrm{MeV}$ in a plasma channel of a few $\mathrm{mm}$.
\end{abstract}

DOI: 10.1103/PhysRevSTAB.5.051301

\section{INTRODUCTION}

In recent years, experiments on the self-modulated laser wakefield (SMLW) accelerator [1] have been conducted at various laboratories [2-8]. In these experiments, usually a high-power laser pulse is focused on a gas jet. The laser pulse is sufficiently strong to create a plasma inside the gas jet and to excite a high-amplitude plasma wakefield as a result of self-modulation. Subsequently, electrons from the plasma are trapped and accelerated in this wakefield. The electron bunches produced in these experiments have been characterized by measurements of the energy distribution, the amount of charge, and the angular distribution. Typically, the energy distribution $f(\gamma)$ of SMLW bunches is exponential, $f(\gamma) \propto \exp \left(-\gamma m c^{2} / T_{e}\right)$, with an effective electron temperature $T_{e}$ in the $\mathrm{MeV}$ range, where $\gamma$ is the Lorentz factor of the electrons [2-8]. The bunches contain high charge: up to $8 \mathrm{nC}$ has been reported [6,7]. The opening angles are usually small and decrease with increasing energy: in several experiments, a tightly focused "hot core" of high energy electrons has been found $[3,8]$. Numerical simulations of the acceleration process have been performed with particle-in-cell (PIC) codes $[9,10]$. These simulation results show that, depending on the laser and plasma parameters involved, there are different mechanisms that lead to electron trapping and acceleration. These include direct laser acceleration [9], wave breaking of the Raman forward scattered plasma wave [10], and coupling to Raman backward scattering [11].

In this paper, we propose and investigate a novel twostage laser wakefield accelerator (LWFA). The first stage is a SMLW accelerator, in which electrons are self-trapped and accelerated from the gas jet plasma. The SMLW electron bunch, with the bulk of the electrons at low energy, is then injected into the second stage, which is a channel-guided resonant LWFA. This second acceleration stage, which we call the postacceleration, is studied in detail with fluid and particle simulations, with and without the effects of self-consistent beam loading.
PACS numbers: 41.75.Jv, 29.25.Bx, 29.27.Bd, 52.38.Ph

The SMLW and resonant LWFA regimes are characterized primarily by the plasma density. For the SMLW, the plasma density is relatively high $\left(>10^{19} \mathrm{~cm}^{-3}\right.$, but still below the critical density), such that the laser pulse extends over several plasma waves $\lambda_{p}$ and the laser power $P$ exceeds the critical power for relativistic self-focusing $P_{c}$ [12]. Here $\lambda_{p}=2 \pi / \omega_{p} ; \omega_{p}=\left(4 \pi n_{e} e^{2} / m\right)^{1 / 2}$ is the plasma frequency, $n_{e}$ is the plasma density, $P_{c}[\mathrm{GW}] \simeq$ $17\left(\lambda_{p} / \lambda\right)^{2}, P[\mathrm{GW}] \simeq 21.5\left(a_{0} r_{0} / \lambda\right)^{2}, r_{0}$ is the spot size of the radial laser profile (assumed Gaussian), $a_{0} \simeq 8.5 \times$ $10^{-9} \lambda[\mu \mathrm{m}]\left(I\left[\mathrm{~W} / \mathrm{cm}^{2}\right]\right)^{1 / 2}$ is the laser strength parameter, and $\lambda$ is the laser wavelength. In this regime, the laser pulse is highly unstable to self-modulation [13-15], which drives a plasma wave to sufficiently high amplitudes such that it traps and accelerates electrons from the background plasma.

For the resonant LWFA a lower density $\left(\sim 10^{18} \mathrm{~cm}^{-3}\right)$ is used such that the laser pulse is approximately equal to the plasma wavelength and the plasma wave is driven in a controlled manner, which allows for a controlled acceleration process. However, for the resonant LWFA it is usually not possible to trap background electrons from the plasma and instead the accelerated electron bunch must be externally injected. In this paper we propose to inject the SMLW bunches into a resonant LWFA to exploit the advantages of both schemes. Because the resonant LWFA involves a much larger dephasing distance, and consequently much higher final electron energies, some form of laser pulse guiding [12] is needed. In this report a parabolic plasma channel is assumed to provide this guiding. Such channels have been produced in experiments [16-18].

In the conventional view of resonant LWFA [1], it is assumed that high energy, high quality bunches are produced by first injecting (from an external source) low energy, high quality electron bunches. To maintain low energy spread, the injected bunch must be short compared to the plasma wavelength and it must be injected at the proper wakefield phase $[19,20]$. In the two-stage LWFA considered here, the injected bunch (produced in the first stage by the SMLW) 
is nonideal in the sense that the initial energy spread of the bunch is large and the bunch is not short compared to the plasma wave of the second stage. Nevertheless, during the postacceleration process, a large fraction (40\%) of the injected bunch is trapped and accelerated in the wakefield, resulting in a high energy $(50 \mathrm{MeV})$ bunch with somewhat reduced energy spread $(60 \%)$. Such a high energy bunch is useful for various applications in which a small energy spread is not essential, such as in nuclear activation for novel isotope production.

For the postacceleration simulations, we use a model distribution for the injected SMLW bunch. This model distribution does not directly use data from SMLW experiments or simulations, but it is constructed in such a way that it contains a number of features found in most experiments and simulations. Using this model for the injected bunch, the postacceleration process is studied in detail via simulations based on a 2D code that combines a particle description for the electron bunch with a fluid model for the wakefields, including all beam loading effects. In the Appendix, a brief description of this code is given.

This paper is organized as follows: In Sec. II, a description of the injected bunch model is given. In Sec. III, beam loading effects are discussed in detail. Section IV contains the simulation results of the postacceleration process. In Sec. V we mention nuclear activation experiments as a possible application for accelerated SMLW bunches. Conclusions are offered in Sec. VI.

\section{DESCRIPTION OF INJECTED BUNCH}

For the effective electron temperature $T_{e}$ of the energy distribution a value of $3.4 \mathrm{MeV}$ has been chosen. The longitudinal bunch distribution is shown in Fig. 1, which is a plot of energy versus $t-z / c$. A number of features found in PIC simulations [10] have been included in this distribution. The simulations typically show a microbunching with a period slightly larger than the plasma wavelength. This is due to both the increase of wavelength in the nonlinear

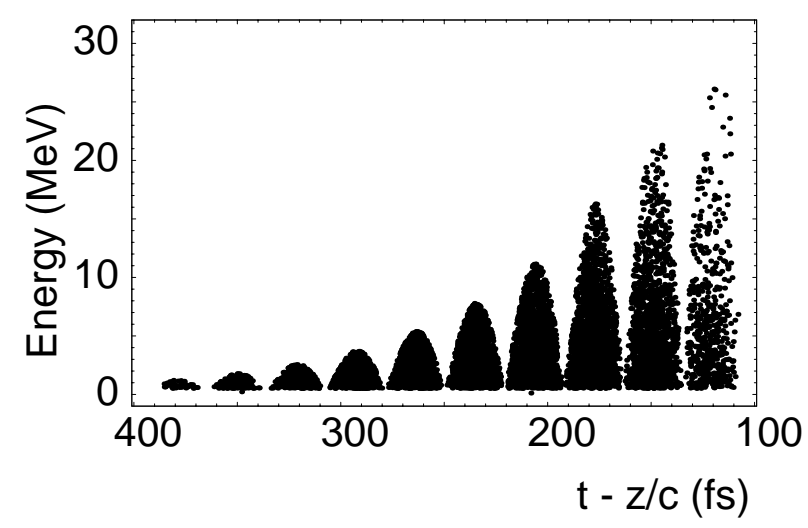

FIG. 1. Model for SMLW bunch: energy vs $t-z / c$.

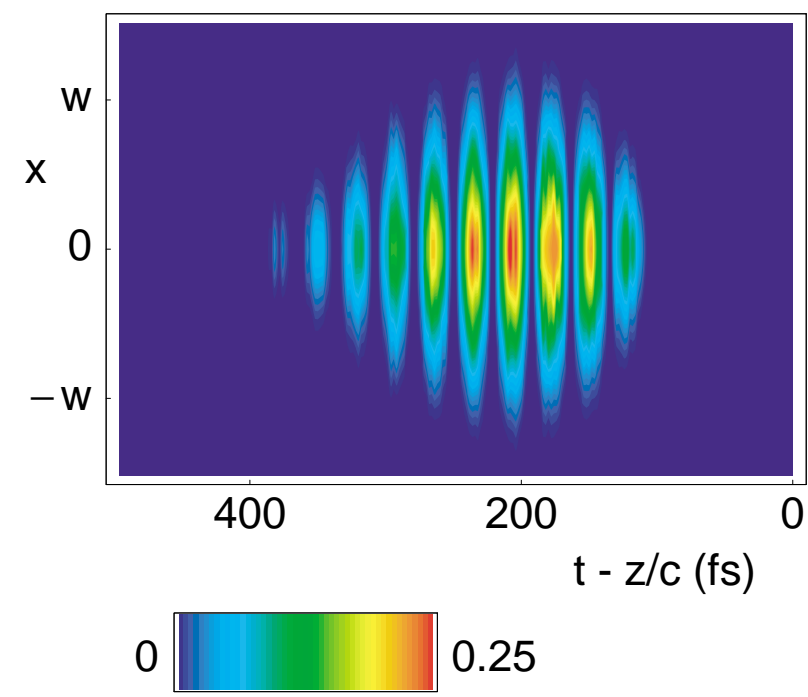

FIG. 2. (Color) Model for SMLW bunch: contour plot of bunch density $n_{b}$ in units of background density $n_{e 0}$ as a function of $x, t-z / c$.

regime and the effect of beam loading (electrons trapped in wakefield buckets modify the wakefield). Also visible in Fig. 1 is a correlation between energy and $t-z / c$, i.e., electrons from the first few wakefield buckets have gained more energy than those trapped in other buckets. This is another beam loading effect also found in PIC simulations.

In Fig. 2, a color contour plot of the bunch density as a function of $x, t-z / c$ is given. The bunch width $w$ is varied in our simulations. The charge of the bunch is varied proportional to $w^{2}$ (this will be explained in Sec. IV) such that the peak density is constant (at about $25 \%$ of the plasma density $n_{e 0}$ on the axis of the plasma channel). In this plot, the microbunching is visible again.

A plot of the assumed opening angle versus energy is given in Fig. 3. This distribution reflects the experimentally observed correlation between the opening angle and energy (smaller opening angle for high-energy electrons).

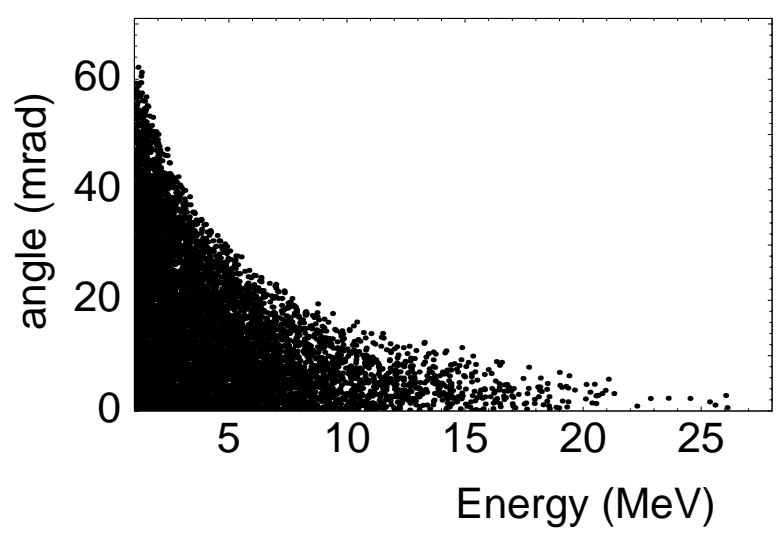

FIG. 3. Model for SMLW bunch: opening angle vs energy. 


\section{BEAM LOADING EFFECTS}

To illustrate the importance of beam loading effects, two runs with the same initial electron distribution are compared: one with beam loading for which all bunch wakefields are included, and a test particle run without beam loading for which no bunch wakefields are calculated. The width of the electron bunch is $w=28 \mu \mathrm{m}$; the charge is $2 \mathrm{nC}$ for the case with beam loading (test particle run is effectively the zero bunch charge limit). Other parameters are plasma density on axis $n_{e 0}=3.4 \times 10^{18} \mathrm{~cm}^{-3}$, laser power $P=40 \mathrm{TW}$, laser spot size $r_{0}=38 \mu \mathrm{m}$, and pulse duration $30 \mathrm{fs}$, which imply a peak laser strength of $a_{0} \simeq 0.9$. The pulse profiles in the axial and transverse directions are assumed to be Gaussian. Furthermore, the laser pulse propagates in a parabolic plasma channel with the channel depth given by the matched beam condition $\Delta n=1 / \pi r_{e} r_{0}^{2} \simeq 7.8 \times 10^{16} \mathrm{~cm}^{-3}$, where $r_{e}$ is the classical electron radius.

Color contours of $F_{x}, F_{z}$ after an acceleration distance of $2 \mathrm{~mm}$ are shown in Figs. 4 and 5. Here, $F_{x}=$
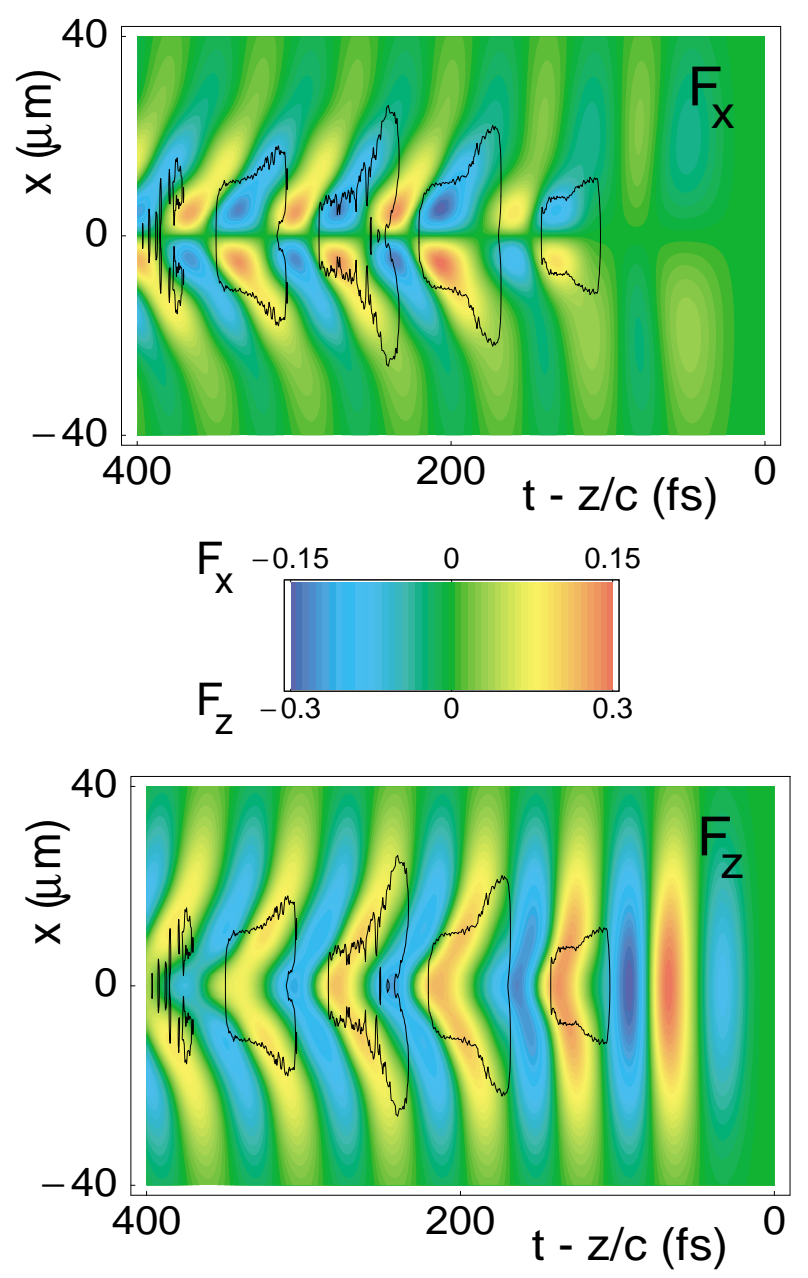

FIG. 4. (Color) Contour plot of $F_{x}, F_{z}$ for a $2 \mathrm{nC}$ bunch at $2 \mathrm{~mm}$ propagation distance. Bunch electron density contours are shown in black.
$-e\left(E_{x}-c B_{y}\right) / m \omega_{p} c, F_{z}=-e E_{z} / m \omega_{p} c$ denote the (normalized) transverse and longitudinal components of the Lorentz force on a relativistic bunch electron. The solid black lines in these figures indicate the electron bunch density contours.

Comparison of the color contours shows the influence of the bunch on the fields. The amplitude of the longitudinal force behind the bunch is considerably lower for the $2 \mathrm{nC}$ bunch (beam loading case, Fig. 4), indicating that the bunch is taking energy from the plasma wave $[21,22]$. Behind the bunch $(t-z / c>350 \mathrm{fs})$ the on-axis accelerating field has been completely canceled by the bunch wakefield. Depending on the position of the electrons in the wakefield, the bunch could also increase the wake amplitude by giving energy to the plasma wave, but inside the bunch, wakefields from the bunch are always decelerating, i.e., the bunch always lowers the value of $F_{z}$. The amplitude of the transverse force behind the bunch is considerably larger for the $2 \mathrm{nC}$ bunch, indicating that the bunch induces strong focusing forces. It is important to note that these forces are always focusing inside the bunch, which is due to a magnetic self-pinching effect known as plasma lensing [23,24].
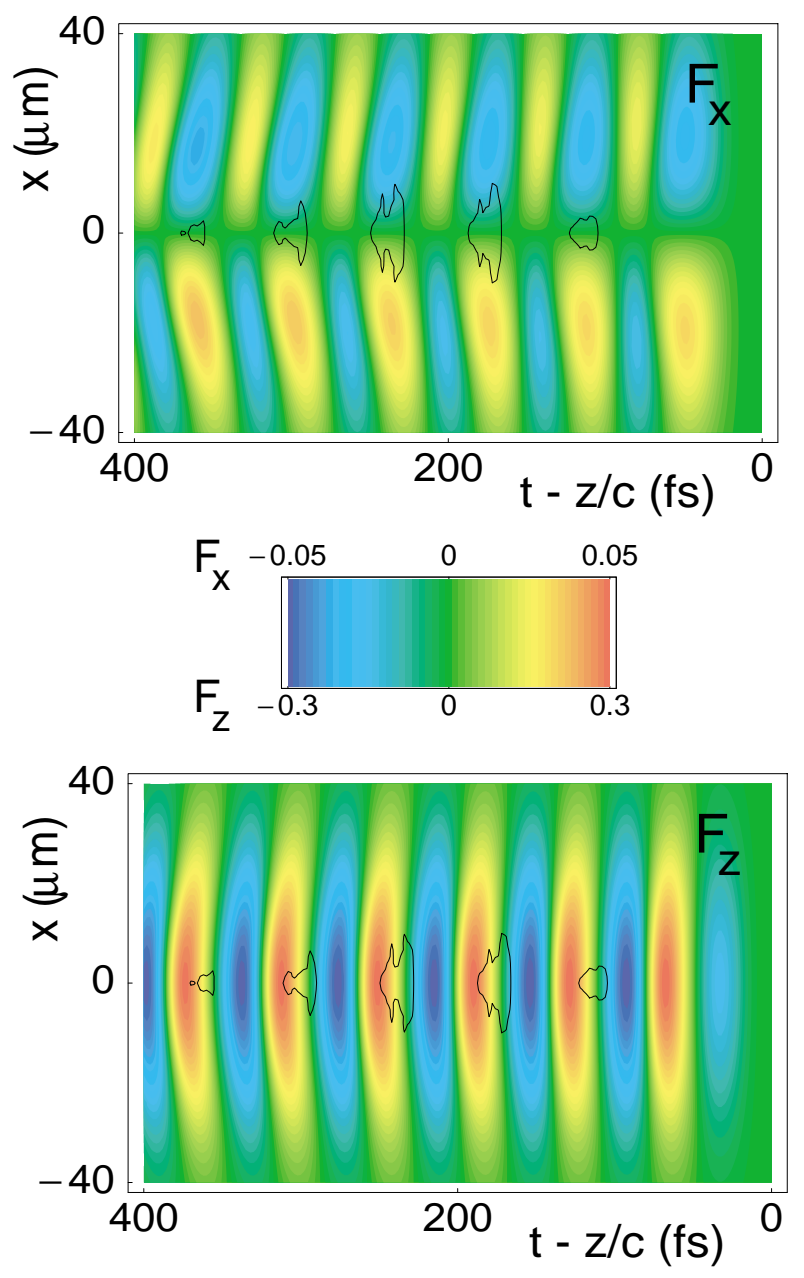

FIG. 5. (Color) As in Fig. 4 for test particle run. 


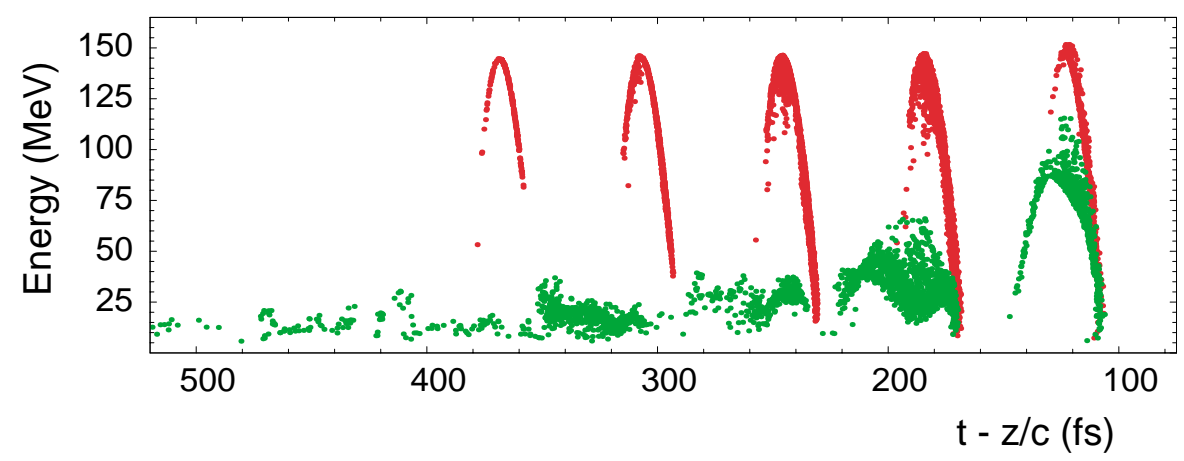

FIG. 6. (Color) Snapshot of energy vs $t-z / c$ at $2.0 \mathrm{~mm}$ for a $2 \mathrm{nC}$ bunch (green) and test particle bunch (red).

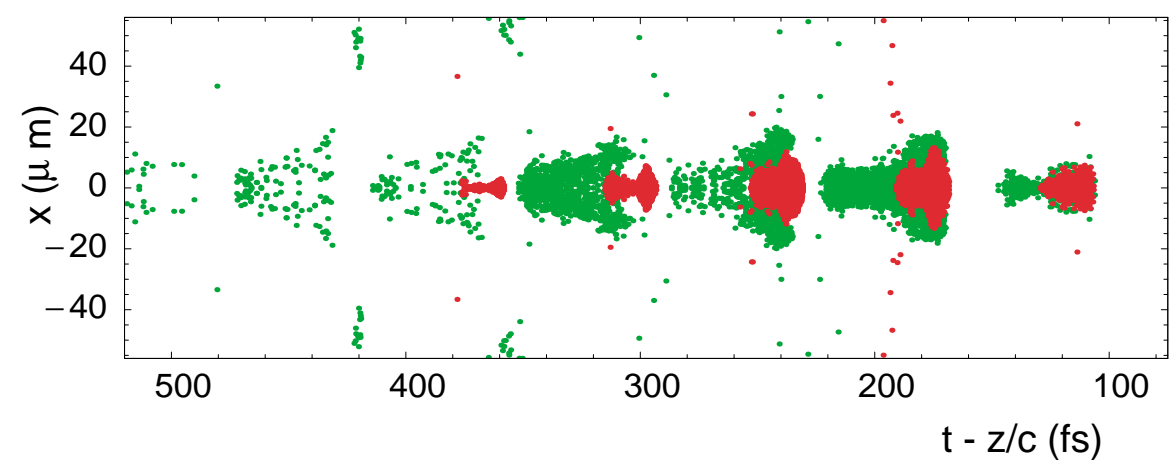

FIG. 7. (Color) Real space snapshot at $2.0 \mathrm{~mm}$ for a $2 \mathrm{nC}$ bunch (green) and test particle bunch (red).

The influence of the bunch wakefields on the electron distribution can be seen from Figs. 6 and 7, which show snapshots of energy versus $t-z / c$ and real space after an acceleration distance of $2 \mathrm{~mm}$. Electrons in the $2 \mathrm{nC}$ bunch have much lower energy than the test electrons, and the difference increases with $t-z / c$. This is due to the asymmetry of the bunch wakefields: particles in the head of the bunch experience only the laser wakefield, and particles in the tail experience both the laser wakefield and the cumulative decelerating wakefield due to all preceding electrons in that bunch.

In the real space snapshot, the distribution of the $2 \mathrm{nC}$ bunch is seen to be rather different from the test particle distribution. Close to the axis, the distribution of the $2 \mathrm{nC}$ bunch is shifted back with respect to the test particle distribution. This is due to the wakefields of the first microbunches, which cause a phase shift of the wakefield close to the axis, resulting in curvature of the wave fronts, as seen in Fig. 4. The $2 \mathrm{nC}$ distribution also has off-axis lobes, giving a typical $\mathrm{Y}$ shape to the microbunches.

\section{SIMULATION RESULTS}

Our simulation results show that the postacceleration process consists of two steps. In the first step, a large portion of the bunch is blown out radially, and only a fraction of the electrons gets trapped in the laser wakefield. The main reason for the blowout to occur is that the spacing of the electron bunch does not match the wave of the wake- field (this is the wave of the low density LWFA plasma, whereas the bunch is modulated at the plasma wave of the high density SMLW plasma). As a result of this, a part of the bunch is injected in a defocusing phase and will be lost. In the second step, the trapped electrons are focused and accelerated.

A formal definition of trapping can be given using the separatrix of a wakefield Hamiltonian [25]. In our case however, this separatrix would be difficult to define because of beam loading effects. Instead we use the following heuristic definition of trapping: a simulation particle is considered to be trapped if it is inside the channel at less than 1.5 laser spot size from the propagation axis. We expect this definition to coincide with the formal one in the second step of the acceleration, after the radial blowout

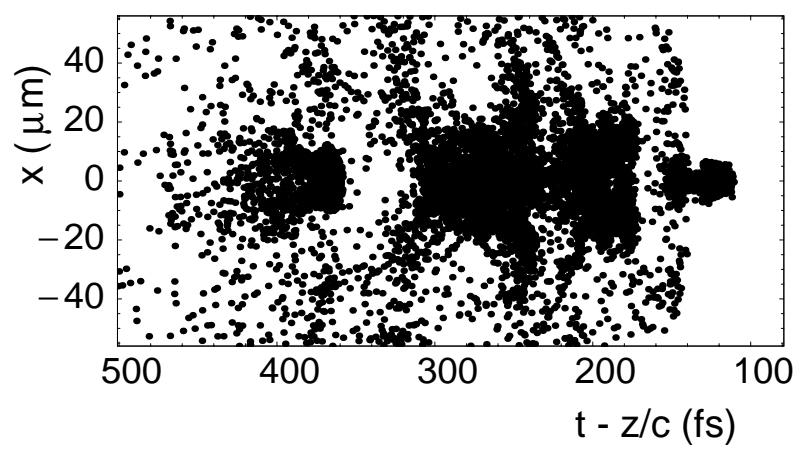

FIG. 8. Real space snapshot at $0.2 \mathrm{~mm}$ for a $2 \mathrm{nC}$ bunch. 


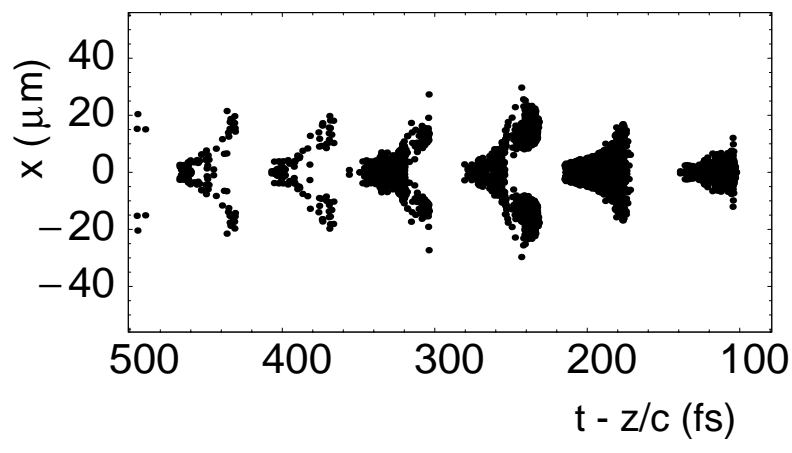

FIG. 9. Real space snapshot at $3.0 \mathrm{~mm}$ for a $2 \mathrm{nC}$ bunch.

of electrons in defocusing regions. Only trapped particles are taken into account for calculating dynamical quantities such as the energy spread and the transverse emittance.

The difference between the acceleration steps is illustrated in Figs. 8 and 9. These figures show snapshots of real space for the $2 \mathrm{nC}$ bunch at propagation distances of 0.2 and $3.0 \mathrm{~mm}$, respectively. Simulation parameters are the same as in Sec. III. Figure 8 shows a typical snapshot of the first step, in which a lot of electrons are being expelled from the channel (the figure shows the region of trapped particles). This is in contrast with a typical snapshot of the second step (Fig. 9), in which the remaining trapped electrons are well within the channel. In this snapshot, the typical Y shape of the microbunches is visible.

In principle, the amount of trapped charge can be increased by increasing the laser power. We have simulated a range of peak powers $P$ between 10 and 100 TW by changing the spot size of the laser pulse proportional to $P^{1 / 2}$ between 19 and $60 \mu \mathrm{m}$ and keeping the amplitude constant (at 0.9 in standard dimensionless units). In the linear wakefield regime (amplitude $\ll 1$ ), this scaling would result in a laser wakefield potential with the same amplitude and a width proportional to $P^{1 / 2}$, which yields an acceptance (amount of charge that can be accelerated) proportional to $P$. To check whether the scaling holds for

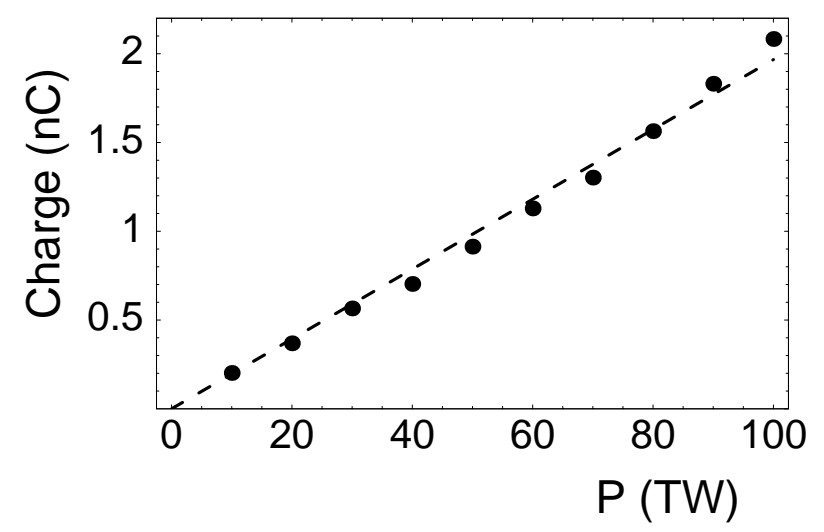

FIG. 10. Trapped charge after $2.7 \mathrm{~mm}$ acceleration for different bunches (see text), as a function of peak power. Dots are simulation results; the dashed line is a linear fit through $(0,0)$.

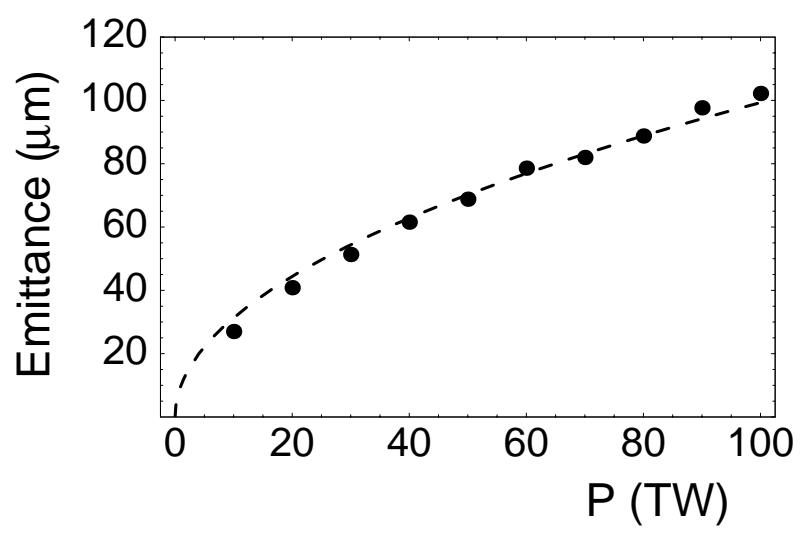

FIG. 11. Normalized transverse emittance after $2.7 \mathrm{~mm}$ acceleration for different bunches (see text), as a function of peak power. Dots are simulation results; the dashed line is a $P^{1 / 2}$ fit.

our parameters, the injected charge has been taken proportional to $P$ by scaling the bunch width $w$ proportional to $P^{1 / 2}$ and keeping the peak density constant. In the linear wakefield regime, this would result in a bunch wakefield potential with the same scaling as the laser wakefield potential, as long as the bunch width $w$ is large compared to $\lambda_{p}$ (quasi-1D regime). For small $w$, the amplitude of the bunch wakefield is reduced as compared to the 1D case [26]. Other parameters are not varied and taken as in Sec. III. In particular, the opening angles are not varied, so the transverse emittance also scales with $P^{1 / 2}$.

Figures 10-12 show trapped charge, normalized emittance, and average energy after an acceleration distance of $2.7 \mathrm{~mm}$, as a function of peak power. The trapped charge scales linearly with $P$, indicating that the trapped fraction is constant. The emittance at $2.7 \mathrm{~mm}$ is proportional to $P^{1 / 2}$ and therefore proportional to the initial emittance. This means that the transverse dynamics is qualitatively the same. The average energy shows a slight decrease with $P$, which is a result of the reduction of the decelerating bunch wakefield for narrow bunches. These results show that the

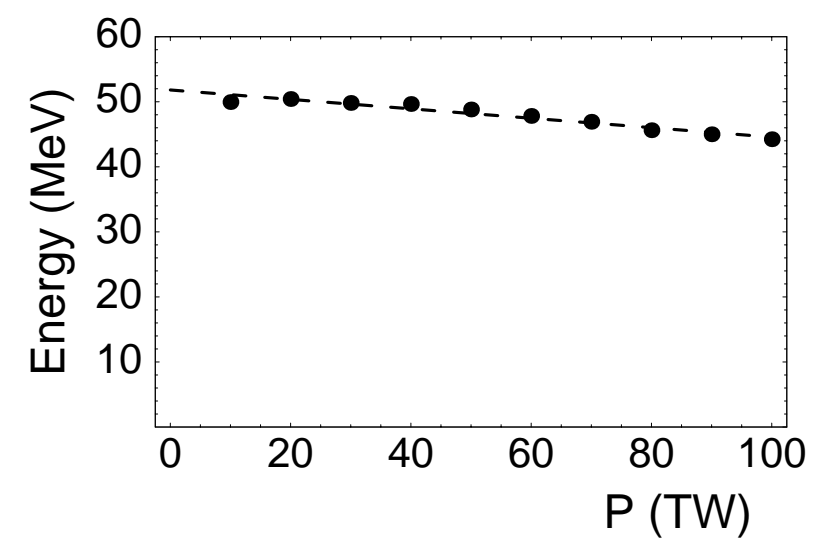

FIG. 12. Average energy after $2.7 \mathrm{~mm}$ acceleration for different bunches (see text), as a function of peak power. Dots are simulation results; the dashed line is a linear fit. 


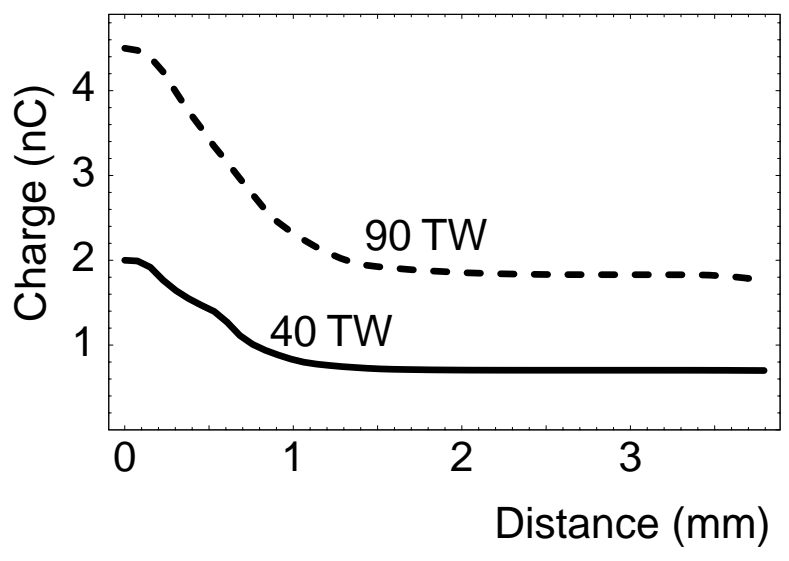

FIG. 13. Trapped charge as a function of propagation distance for the $40 \mathrm{TW}$ case and the $90 \mathrm{TW}$ case.

influence of wakefield nonlinearities is small for the parameters of our simulations, in spite of a relatively high wakefield amplitude.

More details of the acceleration process are given in Fig. 13, which shows the trapped charge as a function of the propagation distance, for both the $40 \mathrm{TW}$ case and the $90 \mathrm{TW}$ case. In the first step $(0-1.5 \mathrm{~mm})$, the trapped fraction is seen to drop quickly to about $40 \%$. In the second step, the trapped fraction remains constant. For the 90 TW case, a slight decrease of the trapped fraction is seen to occur around $3.5 \mathrm{~mm}$, indicating that there is a small amount of additional particle loss.

The plot of normalized transverse emittance as a function of propagation distance, shown in Fig. 14, also illustrates the difference between the steps. In the first step, the emittance rapidly increases, reaches a maximum, and decreases again. This behavior is caused by radial blowout of electrons: while still inside the trapping region, these electrons cause the emittance to grow rapidly. Once they have left the trapping region, they are not counted anymore, and therefore the emittance drops again. In the second step, when the bunch is much more stable inside the

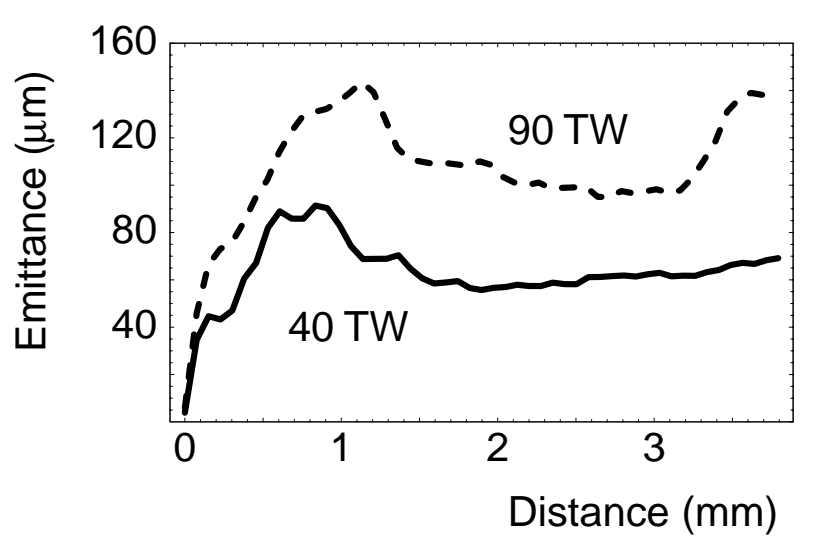

FIG. 14. Normalized transverse emittance as a function of propagation distance for the $40 \mathrm{TW}$ case and the $90 \mathrm{TW}$ case.

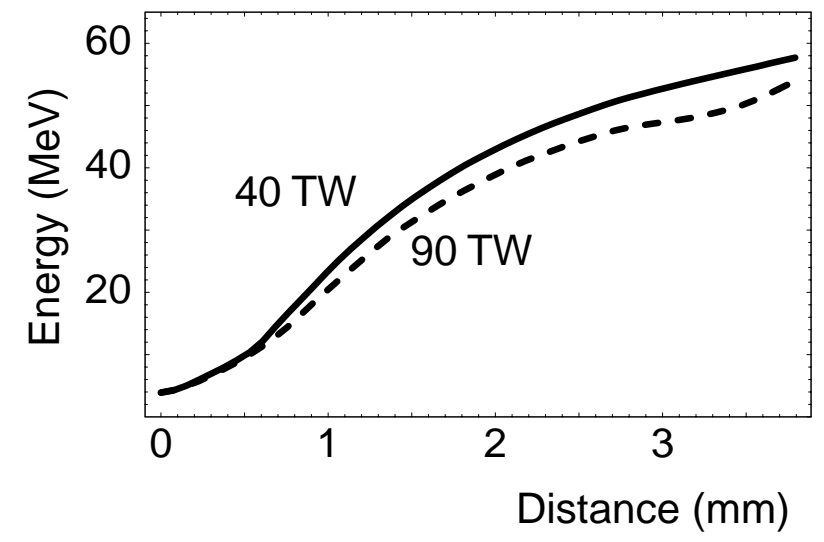

FIG. 15. Average energy as a function of propagation distance for the $40 \mathrm{TW}$ case and the $90 \mathrm{TW}$ case.

channel, the emittance evolves very slowly. For the 90 TW case, Fig. 14 shows additional emittance increase, due to the minor additional particle loss (as seen in Fig. 13).

A plot of average energy as a function of acceleration distance is given in Fig. 15. The energy increase is due to both acceleration and loss of low-energy particles.

Figure 16 shows the relative energy spread as a function of propagation distance. The 40 and 90 TW cases are seen to give almost the same result. A minimum in energy spread of about $60 \%$ occurs at $1.7 \mathrm{~mm}$ acceleration distance.

In Fig. 17 the energy distributions of trapped particles are shown, both at injection (green curve) and after $1.7 \mathrm{~mm}$ acceleration for the $40 \mathrm{TW}$ case (red curve) and the $90 \mathrm{TW}$ case (blue curve). The distribution is seen to change from exponential to highly nonthermal with a peak around $30 \mathrm{MeV}$. Above about $15 \mathrm{MeV}$, the amount of charge in a given energy bin is higher for the accelerated bunches. Around $30 \mathrm{MeV}$, the difference is already 2 orders of magnitude. The blue and red curves almost overlap, demonstrating that in both cases the acceleration dynamics is almost the same. The overlap is a result of normalizing

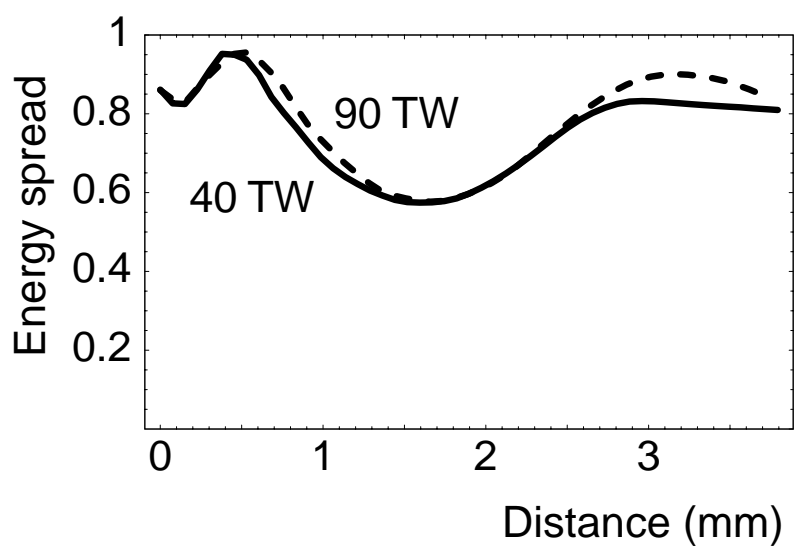

FIG. 16. Relative energy spread as a function of propagation distance for the $40 \mathrm{TW}$ case and the $90 \mathrm{TW}$ case. 


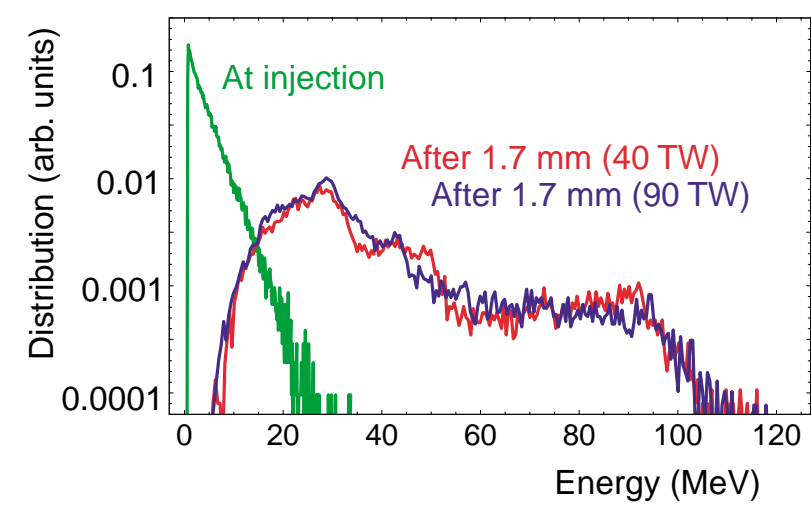

FIG. 17. (Color) Energy distributions of bunches at injection (green) and after $1.7 \mathrm{~mm}$ acceleration for the $40 \mathrm{TW}$ case (red) and the $90 \mathrm{TW}$ case (blue). The total charge for the $90 \mathrm{TW}$ case is $9 / 4$ larger.

the spectra: for the 90 TW case, the bunch charge is of course $9 / 4$ times higher.

\section{NUCLEAR ACTIVATION EXPERIMENTS}

Nuclear activation experiments are a possible application for SMLW generated electron bunches [6,27]. In these experiments, the SMLW bunches interact with a suitable target to produce bremsstrahlung photons. The photons may be used to perform activation experiments involving $(\gamma, n)$ and $(\gamma$, multi- $n)$ reactions. The use of SMLW bunches is not particularly efficient because typically only a small fraction of the electrons has sufficiently high energy to produce the energetic photons necessary for the $(\gamma, n)$ and $(\gamma$, multi- $n)$ reactions. The yield $Y$ in an activation experiment can be calculated from the scattering cross section $\sigma$ as

$$
Y=\int d \omega \sigma(\omega) \frac{d N_{\gamma}}{d \omega},
$$

where $d N_{\gamma} / d \omega$ is the photon number spectrum produced by bremsstrahlung. Note that the scattering cross section $\sigma$ usually has a single peak around the resonant energy, typically a few $10 \mathrm{~s}$ of $\mathrm{MeV}$. This suggests that the yield could be greatly improved if the bulk of the SMLW-bunch electrons is accelerated to higher energy by a resonant LWFA, as discussed in Sec. IV. Figure 17 indicates that the number of electrons available to produce photons at the resonant energy of the reaction cross section can be orders of magnitude higher for the postaccelerated beam.

As a numerical example, consider activation of ${ }^{63} \mathrm{Cu}$ by the reactions $(\gamma, n)$ and $(\gamma, 2 n)$, with the reaction energy thresholds 10.8 and $19.7 \mathrm{MeV}$, respectively [6]. Consider production of bremsstrahlung photons using a simplified model based on a thin target approximation (i.e., much less than the radiation length), such that a relativistic electron experiences only a single scattering event and multiple scattering and cascade effects are neglected. For a single highly relativistic electron, the bremsstrahlung pho- ton spectrum [28] is approximately $\left[d N_{\gamma} / d \omega\right]_{1}=c_{\gamma} / \omega$ for $\hbar \omega<\gamma m c^{2}$, where $c_{\gamma}$ is a constant determined by the target properties. The bremsstrahlung spectrum produced by an electron bunch with energy distribution $f(\gamma)$ is

$$
\frac{d N_{\gamma}}{d \omega}=\frac{c_{\gamma}}{\omega} \int_{\hbar \omega / m c^{2}}^{\infty} d \gamma f(\gamma)
$$

and therefore the yield produced by the bunch is

$$
Y=c_{\gamma} \int d \omega \frac{\sigma(\omega)}{\omega} \int_{\hbar \omega / m c^{2}}^{\infty} d \gamma f(\gamma) .
$$

We can compare the activation $Y_{1}$ produced by the SMLW electron bunch distribution $f_{1}$ and the activation $Y_{2}$ produced by the postaccelerated electron bunch $f_{2}$ shown in Fig. 17. Evaluating Eq. (3) for the distributions shown in Fig. 17 we find $Y_{2} / Y_{1} \simeq 40$ for the reaction ${ }^{63} \mathrm{Cu}(\gamma, n)$, and $Y_{2} / Y_{1} \simeq 4.2 \times 10^{2}$ for the reaction ${ }^{63} \mathrm{Cu}(\gamma, 2 n)$. As the numerical example shows, orders of magnitude improvement can be achieved in nuclear activation yields by postaccelerating a SMLW bunch using a two-stage laser wakefield accelerator. More detailed simulations using a Monte Carlo based nuclear interaction code [29] have been carried out to design optimized bremsstrahlung targets for enhancing the yield of specific nuclear reactions [30]. These calculations confirm that up to 4 orders of magnitude increase in activation yields can be obtained using beams from a channel guided laser wakefield accelerator as opposed to the SMLW bunch.

\section{CONCLUSIONS}

We have proposed and investigated a novel two-stage LWFA accelerator consisting of a SMLW (stage 1) that generates a low-energy electron bunch, which is injected into a channel-guided resonant LWFA (stage 2) for acceleration to high energy. The postacceleration process (stage 2 ) was simulated in detail using a fluid model for the plasma and a particle method for the bunch electrons that includes the effects of beam loading. Because of the lower plasma density involved, resonant LWFA offers a larger dephasing energy; because the dephasing distance is longer, a plasma channel is needed for pulse guiding. We have simulated trapping of about $40 \%$ of the injected charge and acceleration to an average energy of about $50 \mathrm{MeV}$ for a density of $3.4 \times 10^{18} \mathrm{~cm}^{-3}$ in a plasma channel of $3 \mathrm{~mm}$. We have illustrated the importance of beam loading effects by comparing the run of a $2 \mathrm{nC}$ bunch with a test particle run.

Our simulation results show that the postacceleration process consists of two steps: in the first step there is radial blowout of electrons that are injected in defocusing regions; in the second step the remaining electrons are focused and accelerated. The energy distribution changes from an exponential distribution to a highly nonthermal distribution with somewhat reduced energy spread (about 60\%).

We have investigated a range of peak powers between 10 and $100 \mathrm{TW}$ for the laser pulse by scaling the spot 
size between 19 and $60 \mu \mathrm{m}$ and keeping the peak amplitude constant. Scaling bunch width and bunch charge accordingly, we have found that the dynamics was about the same for all simulations. From this we conclude that a quasi-1D linear scaling holds for our parameters, in spite of a relatively high wakefield amplitude and a relatively small bunch width for the low-power cases.

As a possible application for the bunch after postacceleration we have mentioned nuclear activation experiments, in which a small electron energy spread is not necessary. From our simulation results, the nuclear activation yield for accelerated bunches is estimated to be orders of magnitude higher than for the injected SMLW bunches.

\section{ACKNOWLEDGMENTS}

One of the authors (A. J. W. R.) gratefully acknowledges the hospitality of Lawrence Berkeley Laboratory and financial support from CPS (Dutch Research School for Plasma Physics and Radiation Technology). This work was supported by the U.S. Department of Energy, Office of High Energy Physics and Office of Biological and Environmental Research, Contract No. DE-AC-03-76SF0098.

\section{APPENDIX: DESCRIPTION OF THE SIMULATION CODE}

In our simulation code, a PIC model for the electron bunch is combined with the electron fluid description for the plasma response. It is a 2D slab geometry code, developed at Eindhoven University of Technology. It has been used to describe the laser wakefield acceleration of ultrashort electron bunches with high beam loading [19].

The quasistatic approximation $[1,12,31]$ is used for calculating the wakefields. In the wakefield calculation, a nonevolving laser pulse is assumed, and therefore effects such as pump depletion, pulse broadening, and frequency redshifting are neglected. The hydrodynamic wakefield equations are fully nonlinear. In the absence of beam loading, the plasma response is given by the $2 \mathrm{D}$ slab version of the quasistatic equations given in Refs. [1,31]. All beam loading effects are taken into account by including the bunch density of accelerated electrons as a source term for the wakefields.

[1] E. Esarey, P. Sprangle, J. Krall, and A. Ting, IEEE Trans. Plasma Sci. 24, 252 (1996).

[2] A. Modena, Z. Najmudin, A. E. Dangor, C. E. Clayton, K. A. Marsh, C. Joshi, C. B. Darrow, C. Danson, D. Neely, and F. N. Walsh, Nature (London) 377, 606 (1995).

[3] D. Umstadter, S.-Y. Chen, A. Maksimchuk, G. Mourou, and R. Wagner, Science 273, 472 (1996); R. Wagner, S.-Y. Chen, A. Maksimchuk, and D. Umstadter, Phys. Rev. Lett. 78, 3125 (1997); S.-Y. Chen, M. Krishnan, A. Maksimchuk, R. Wagner, and D. Umstadter, Phys. Plasmas 6, 4739 (1999).
[4] A. Ting, C. I. Moore, K. Krushelnick, C. Manka, E. Esarey, P. Sprangle, R. Hubbard, H. R. Burris, and M. Baine, Phys. Plasmas 4, 1889 (1997); C. I. Moore, A. Ting, K. Krushelnick, E. Esarey, R. F. Hubbard, H. R. Burris, C. Manka, and P. Sprangle, Phys. Rev. Lett. 79, 3909 (1997).

[5] D. Gordon, K.-C. Tzeng, C. E. Clayton, A. E. Dangor, V. Malka, K. A. Marsh, A. Modena, W. B. Mori, P. Muggli, Z. Najmudin, D. Neely, C. Danson, and C. Joshi, Phys. Rev. Lett. 80, 2133 (1998).

[6] W. P. Leemans, D. Rodgers, P. E. Catravas, C. G. R. Geddes, G. Fubiani, E. Esarey, B. A. Shadwick, R. Donahue, and A. Smith, Phys. Plasmas 8, 2510 (2001).

[7] V. Malka, J. Faure, J. R. Marquès, F. Amiranoff, J. P. Rousseau, S. Ranc, J.P. Chambaret, Z. Najmudin, B. Walton, P. Mora, and A. Solodov, Phys. Plasmas 8, 2605 (2001).

[8] C. Gahn, G. D. Tsakiris, A. Pukhov, J. Meyer-ter-Vehn, G. Pretzler, P. Thirolf, D. Habs, and K. J. Witte, Phys. Rev. Lett. 83, 4772 (1999); G. D. Tsakiris, C. Gahn, and V. K. Tripathi, Phys. Plasmas 7, 3017 (2000).

[9] A. Pukhov, Z.-M. Sheng, and J. Meyer-ter-Vehn, Phys. Plasmas 6, 2847 (1999).

[10] K.-C. Tzeng, W. B. Mori, and T. Katsouleas, Phys. Rev. Lett. 79, 5258 (1997); Phys. Plasmas 6, 2105 (1999).

[11] E. Esarey, B. Hafizi, R. Hubbard, and A. Ting, Phys. Rev. Lett. 80, 5552 (1998).

[12] E. Esarey, P. Sprangle, J. Krall, A. Ting, and G. Joyce, Phys. Fluids B 5, 2690 (1993); E. Esarey, P. Sprangle, J. Krall, and A. Ting, IEEE J. Quantum Electron. 33, 1879 (1997).

[13] E. Esarey, J. Krall, and P. Sprangle, Phys. Rev. Lett. 72, 2887 (1994).

[14] W. B. Mori, C. D. Decker, D. E. Hinkel, and T. Katsouleas, Phys. Rev. Lett. 72, 1482 (1994).

[15] N.E. Andreev, L. M. Gorbunov, V.I. Kirsanov, A. A. Pogosova, and R. R. Ramazashvili, Phys. Scr. 49, 101 (1994).

[16] H. M. Milchberg, T. R. Clark, C. G. Durfee, T. M. Antonsen, and P. Mora, Phys. Plasmas 3, 2149 (1996).

[17] Y. Ehrlich, C. Cohen, A. Zigler, J. Krall, P. Sprangle, and E. Esarey, Phys. Rev. Lett. 77, 4186 (1996).

[18] P. Volfbeyn, E. Esarey, and W. P. Leemans, Phys. Plasmas 6, 2269 (1999).

[19] A. J. W. Reitsma, V. V. Goloviznin, L. P. J. Kamp, and T. J. Schep, Phys. Rev. E 63, 046502 (2001).

[20] N. E. Andreev, S. V. Kuznetsov, and I. V. Pogorelsky, Phys. Rev. ST Accel. Beams 3, 021301 (2000).

[21] S. Wilks, T. Katsouleas, J. M. Dawson, P. Chen, and J. J. Su, IEEE Trans. Plasma Sci. 15, 210 (1987).

[22] A. J. W. Reitsma, R. M. G. M. Trines, and V. V. Goloviznin, IEEE Trans. Plasma Sci. 28, 1165 (2000).

[23] R. Govil, W.P. Leemans, E. Yu. Backhaus, and J.S. Wurtele, Phys. Rev. Lett. 83, 3202 (1999).

[24] J. S. T. Ng, P. Chen, H. Baldis, P. Bolton, D. Cline, W. Craddock, C. Crawford, F. J. Decker, C. Field, Y. Fukui, V. Kumar, R. Iverson, F. King, R. E. Kirby, K. Nakajima, R. Noble, A. Ogata, P. Raimondi, D. Walz, and A. W. Weidemann, Phys. Rev. Lett. 87, 244801 (2001).

[25] E. Esarey and M. Pilloff, Phys. Plasmas 2, 1432 (1995).

[26] R. Keinigs and M. Jones, Phys. Fluids 30, 252 (1987); A. G. Khachatryan, Phys. Rev. E 65, 046504 (2002). 
[27] M. I. K. Santala, M. Zepf, F. N. Beg, E. L. Clark, A.E. Dangor, K. Krushelnick, M. Tatarakis, I. Watts, K. W. D. Ledingham, T. McCanny, I. Spencer, A. C. Machacek, R. Allott, R. J. Clarke, and P. A. Norreys, Appl. Phys. Lett. 78, 19 (2001).

[28] J.D. Jackson, Classical Electrodynamics (Wiley, New
York, 1975).

[29] J. F. Briesmeister, Los Alamos National Laboratory Report No. LA-13709-M, 2000.

[30] R. Donahue (private communication).

[31] J. Krall, A. Ting, E. Esarey, and P. Sprangle, Phys. Rev. E 48, 2157 (1993). 